\title{
A Chronic Disease Exercise Rehabilitation System Based on Cloud Computing Architecture
}

\author{
Yongliang Zhang, ${ }^{1,2}$, Jun $\mathrm{Ye}^{2}$, Jian Yang ${ }^{2}$, Qiao Guo ${ }^{2}$, Yining Sun², Weimin Wang ${ }^{1}$ and Qiang Zeng ${ }^{1 *}$ \\ ${ }^{1}$ Institute of Health Management, Chinese PLA General Hospital, Beijing, China \\ ${ }^{2}$ Research Center for Information Technology of Sports and Health, Institute of Intelligent Machines Chinese Academy of \\ Sciences, Hefei, China \\ ${ }^{*}$ Corresponding author
}

\begin{abstract}
In view of the current status of chronic disease management development and the current society's need for intelligent exercise monitoring, this article has conducted research on the rehabilitation system and key technologies for its realization. First, this article uses a cloud computing architecture to develop a chronic disease rehabilitation system. Secondly, based on the mobile phone system, this article has developed health-care and client terminal that can be remotely connected to the system. Finally, this article applies the personalize exercise prescription reasoning algorithm and exercise effect evaluation algorithm to the developing of the project. The system designed in this article has functions such as automatic reasoning of exercise prescriptions, remote monitoring of exercise, and evaluation of exercise effects. Tests have shown that the system operates stably and can achieve the purpose of closed-loop chronic disease management with exercise as the core.
\end{abstract}

Keywords-mobile terminal; cloud computing; exercise intervention; chronic disease management

\section{INTRODUCTION}

This template, In recent years, the rapid development of cloud computing technology has injected new vitality into the development and the realization of medical informatization .Cloud computing uses the internet as a carrier to provide dynamic, scalable, flexible services and storage resource through the "click-and-play" approach, which helps to solve the drawbacks of the traditional organization-based health-care informationization.

Chronic disease management refers to the provision of a comprehensive, active, and effective management of patients with chronic diseases, so that patients with chronic diseases can get better treatment to promote their recovery, reduce the incidence of complications and the patients burden caused by the disease and improve the quality of life[1]. This article uses cloud computing in conjunction with mobile terminals to use exercise as the main intervention for chronic disease management. By creating closed-loop management services for precise exercise and remote tracking, it will integrate medical resources and effectively solve current problems of chronic disease management, at the same time, it will expand the radius of medical services and strengthen the development of the health behavior of patients through continuous tracking and management.

\section{Cloud COMPUting ARCHITECTURE PlatForm OVERVIEW}

\section{A. Cloud Computing Technology}

Cloud computing is a new technology that is currently developing. Through the analysis of the current cloud platform markets, the author thinks that Microsoft's Azure cloud platform is relatively clear[3,12], the open source AbiCloud cloud platform is relative complete[4], and Hadoop under Apache is widely supported[5]. Therefore, we have made full use of their advantages in designing cloud computing platforms, and finally completed system development using Alibabba Cloud Computing Platform.

\section{B. Service-Oriented Technology}

Considering the flexibility and scalability requirement of the platform, this system takes advantage of the CXF framework's ability to distribute and deploy independently or distributedly[6], the ability of Spring DM to be dynamically modularized, and Tuscany's architecture support for system integration[7,8]. Based on CXF+Spring DM and Tuscany, together with the business service package design, the service platform is implemented to provide basic service support for the implementation of the application platform.

\section{Platform Framework}

The J2EE architecture is an open enterprise-level application specification defined by SUN Company. It provides a multilevel distributed application and a series of development specifications[9]. The J2EE architecture provides the middletier integration framework to meet the needs of applications that require high availability without much expense. By providing a unified development platform, J2EE reduces the cost and complexity of developing mutli-tier applications, while providing strong support for the integration of existing application.

The distributed architecture in J2EE architecture design can be roughly divided into single-level structure, 2-level structure, 3-level structure and multi-level structure. The multi-level structure provides a good mechanism for building a system that is scalable, flexible, and easy to maintain[10]. The technical framework of this application platform introduces technologies such as Service-Oriented Architecture and Open Service 
Gateway Initiative on the basis of absorbing the N-tier distributed architecture of the J2EE architecture, which further improves the standardization and scalability of the platform technology.

\section{Distributed Cache Technology}

The distributed cache has the characteristics of high performance, dynamic scalability, ease of use, and high availability[11].

\section{E. Distributed Data}

Although cloud computing platforms also support distributed data, considering the flexible deployment requirement of the system platform, it is also necessary to support distributed databases at the application platform level in the absence of cloud computing deployment. In this regard, the system adopts such a strategy:

- Simple design application: Use Java DataBase Connectivity-encapsulated Plain Ordinary Java Object directly, and use a common Data Access Object (DAO) framework to implement database operations.

- Object-oriented design applications: Use Object Relational Mapping (ORM) encapsulation, and a common DAO framework to achieve the operation of the database.

- Large-scale integrated application: Combine Java DataBase Connectivity (JDBC) and ORM frameworks, through the integration of open source Guzz distributed

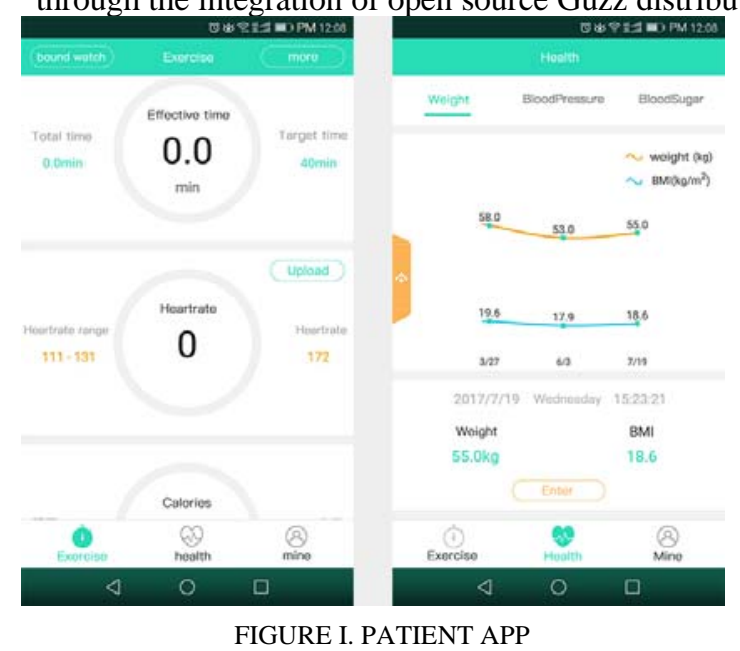

The management platform of the medical platform mainly includes rehabilitation program platform and user tracking platform. The major functions of the rehabilitation program platform include user registration, medical archiving, questionnaire survey, physical examination equipment docking data access framework, supporting distributed data operations of large-scale systems.

- Integrated applications: Consider compatibility with the Service Component Architecture (SCA) service framework Tuscany. This section uses Service Data Objects (SDO) to encapsulate data, and a common DAO framework to implement database operations.

\section{THE OVERALL ARCHITECTURE OF CHRONIC DISEASE EXERCISE REHABILITATION SYSTEM}

There are three layers of the chronic disease exercise rehabilitation system, which are the mobile terminal application layer, medical platform management layer and cloud service layer.

Mobile terminal application layer includes patient APP and health-care APP. In order to meet different models of mobile phones in the market, the APP of this project has been developed based on Android and IOS platforms respectively. The main functions of patient APP include heart rate data upload, daily exercise statistics(exercise time, exercise steps, energy consumption), exercise score, doctor-patient communication, health record management, and physiological index(blood sugar, blood pressure, blood lipids, weight) management. The main functions of the health-care APP include health record query, patient exercise tracking, physiological index tracking, and doctor-patient communication. Patient APP is shown in Figure 1. Health-care APP is shown in Figure 2.

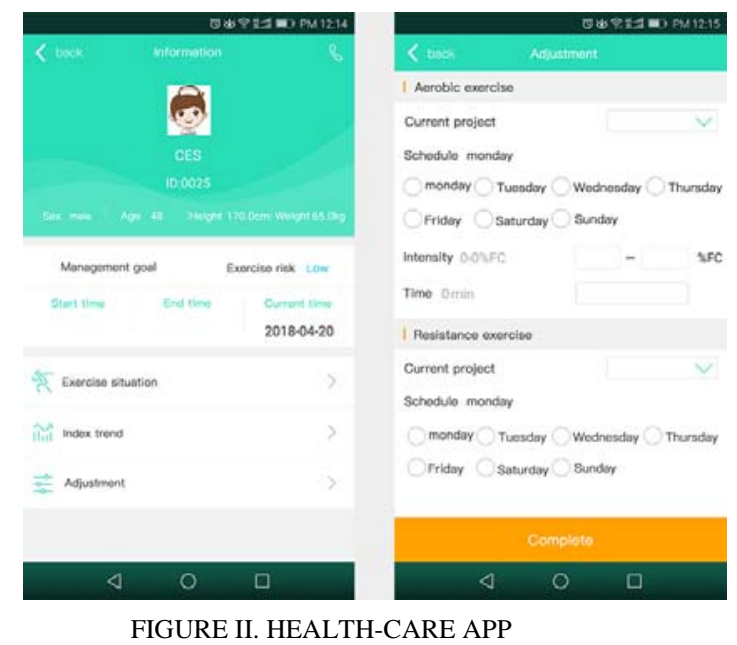

function, exercise prescription inference function. The user tracking platform mainly implements functions such as patient exercise tracking, physiological index tracking, and exercise prescription adjustment. Implementation effects are shown in Figure 3 and Figure 4. 

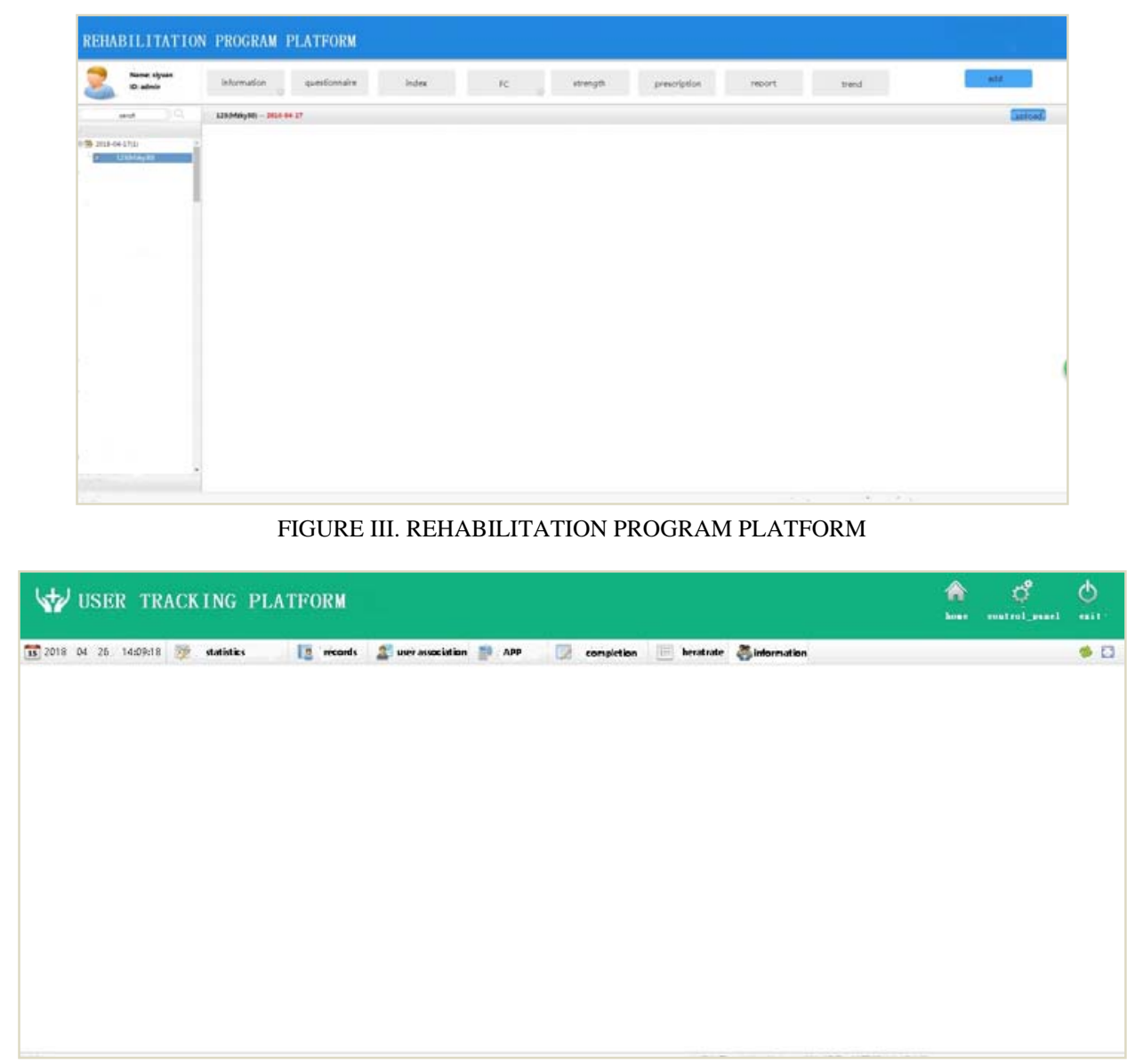

FIGURE IV. USER TRACKING PLATFORM

Cloud service layer provides relevant data processing and technical support for cloud platform, such as the processing of equipment data, providing related computing services, and load balancing. In addition, the cloud service layer also provides virtualization services to hardware and software such as hosts, databases, and networks. RESTful architecture is favored by more and more developers because of its extensibility and simplicity, among which HTTP is a typical application of this architectural style[13]. The system uses meta-operations of data: GET is used to obtain resources, POST is used to create new resources, PUT is used to update resources, DELETE is used to delete resources. What's more, the system uses the standard Bluetooth protocol to implement communication between the mobile terminal and the Bluetooth device. The physical examination device and the system use json communication interface to realize bidirectional transmission of user information and detection information.

\section{THE KEY TECHNOLOGIES OF CHRONIC DISEASE REHABILITATION SYSTEM}

The chronic disease exercise rehabilitation system realizes two main functions of in-hospital prescription generation and out-hospital remote monitoring. As the core technology of the in-hospital, personalized exercise prescription reasoning technology determines patient exercise management goals and exercise prescriptions by integrating basic patient information, physiological indicators, questionnaire information, and cardiopulmonary test information.

\section{A. Personalized Exercise Prescription Reasoning Technology}

The Personalized exercise prescription reasoning techniques include the determination of management goal and the generation of exercise prescriptions.

\section{1) Determine management goals}

Before the exercise prescription is generated, the system confirms exercise taboos by comprehensively understanding the patient's condition. If there are serious complications of diabetes, such as uncontrolled metabolic disease, systolic blood pressure up to $200 \mathrm{mmHg}$ or diastolic blood pressure to $110 \mathrm{mmHg}$ or Acute heart disease[14], patients are forbidden to exercise.

After excluding exercise taboos, the system prioritizes the main diseases, including diabetes, prediabetes, hypertension, prehypertension, obesity, overweight and determines the management goals of exercise interventions base on these diseases.

\section{2) Generate exercise prescription}

Exercise prescription generation depends on management goals, physical activity levels, cardiopulmonary levels and 
exercise habits. The International Physical Activity Questionnaire-Short Form was used to determine the level of physical activity by determining the weekly frequency and daily cumulative time of different intensity activities[15].

We use a treadmill as a carrier to evaluate the patient's cardiopulmonary endurance level by outputting constant power loads and monitoring exercise heart rate. According to the widely accepted theory of indirect load test, the cardiopulmonary endurance level can be calculated[16].

Exercise prescriptions are set in a step-by-step manner which is gradually increasing exercise intensity and time, and is divided into the adaptive exercise prescription and the strength exercise prescription. The adaptive prescription exercise is performed within one month of the start of exercise. This stage is mainly used to train the patient's compliance and adaptability, so the exercise intensity and time are both low. During the period of strength exercise, the body's adaptation to exercise is relatively stable after the end of the adaption exercise period. The effects of exercise on the intervention of chronic diseases can be strengthened by increasing exercise intensity and exercise time.

The exercise intensity of patients with chronic diseases is roughly set as follows: Patients with good cardiopulmonary endurance are higher than patients with low cardiopulmonary endurance; Patients with low exercise risk are higher than those with high exercise risk; Patients with single disease are higher than those with comorbidities.

The type of exercise generates several types of exercise for the patient's selection through the patient's favorite sports and the appropriate types of exercise under different management goals.

\section{B. Exercise Effect Evaluation}

The evaluation of the patient's exercise effect refers to a detailed analysis of the patient's daily exercise to determine whether the exercise is scientific and effective. The system analyzes the data (heart rate, step, calories) collected by the wearable device. Through the establishment of exercise performance evaluation, the subjective feelings of the patient and objective data are used to score the daily exercise status. The subjective feeling refers to the degree of fatigue of the patient after each exercise, such as no feeling, very relaxed, relaxed, a little tired, tired, very tired. The objective data includes the patient's daily effective exercise time, high intensity exercise time, and exercise time that exceeds the maximum heart rate. The establishment of the evaluation system will effectively improve the patient's motivation, and will provide reference for the medical staff's investigation of the patient's exercise compliance.

\section{CONCLUSION}

This article studies and implements a chronic disease exercise rehabilitation system based on cloud computing architecture. We use the "in-hospital assessment and out-ofhospital management" service for intervention to effectively expand the service radius, and strengthen the development of healthy behavior through continuous tracking and management.
In addition, the establishment of personalized exercise prescription reasoning technology and exercise effect evaluation technology will provide theoretical support for creating a closed-loop chronic disease management services.

In the future, the study of chronic disease management will focus on the evaluation model of exercise intervention effects. On the one hand, the model will evaluate the effectiveness of current chronic disease management services, on the other hand, it will promote the current chronic disease management service and will help to optimize and iterate the knowledge base and the service of management. Compared with other intervention services, the intervention services centered on exercise are more likely to form a closed-loop management, which will greatly promote the development of chronic disease management and play a pioneering role in China.

\section{ACKNOWLEDGMENT}

This work was supported by the Science and Technology Service Network Program of Chinese Academy of Sciences under Grant KFJ-STS-ZDTP-033.

\section{REFERENCES}

[1] Changxiu Liang. Application of health management in chronic disease management[J]. Chinese Community Doctors. 2011, 13(4): 211-212.

[2] Tingting Zhang,FengKun Wang,Jing Ge,Tianran Bu.Research on smart medical recommendation system based on cloud platform[J] .Journal of Jiamusi University(Natural Science Edition). 2017, 35(04):671-673.

[3] Ronghua Shi, Yan Yan, Chunhua Peng.Mobile Health System Based on Windows Azure Cloud Computing[J].SAMSON. 2015, 23(05): 37-40.

[4] Min Yang.Design and Implementation of Stochastic Transaction Derivatives Based on Cloud Computing[D]. Fudan University. 2011.

[5] Tianming Pan. The parallel research on decision algorithm based on Hadoop[D]. East China Normal University. 2012.

[6] Bin Hou, Hongbing Tu, Yunfu Wang. CXF framework based configurable enterprise content management platform web service[J]. Telecommunications Science. 2016, 32(05): 191-196.

[7] Jirong Chen, Fangting Yang, Shouyi Zhan. Design and implementation of distributed SCA application based on Tuscany SCA[J]. Journal of Guangxi University: Nat Sci Ed. 2011, 36(06):967-971.

[8] Jingwen Chen, Yang Zhang. Tuscany-based implementation of multiinstance management. Computer engineering \& Software. 2014, 35(09): 26-30.

[9] Kai Lin.Analysis and Design of Office Automation System Based on J2EE Architecture[J]. Computer Knowledge and Technology. 2008, (09):1656-1658+1665.

[10] Yong Zhang.Research and Development of Enterprise Personnel Management System Based on J2EE Architecture[D]. University of Electronic Science And Technology Of China. 2013.

[11] Xiuqing Feng.Summary based on Distributed Cache Technology[J].Electronics World,2017(15):87.

[12] Calder B, Wang J, Ogus A, et al. Windows Azure Storage: a highly available cloud storage service with strong consistency[C]. ACM Symposium on Operating Systems Principles. 2011:143-157.

[13] Mingwei Tang, Yijie Bian, Feifei Tao. Research and Implementation of Library Management System under RESTful Architecture[J].Data Analysis and Knowledge Discovery. 2010, 26(9): 84-89.

[14] Gibbons R J, Balady G J, Bricker J T, et al. ACC/AHA 2002 guideline update for exercise testing[J]. Journal of the American College of Cardiology. 2002, 40(8).

[15] Grimm E K, Swartz A M, Hart T, et al. Comparison of the IPAQ-Short Form and accelerometry predictions of physical activity in older adults.[J].Journal of Aging and Physical Activity. 2012, 20(1): 64-79. 
[16] Hang Xu.Treadmill-based cardiopulmonary endurance test and training method[D]. University Of Science and Technology of China. 2014. 